\title{
Caregivers' Use of Patient Portals: Findings from a 2019 National Survey
}

J Gen Intern Med 36(10):3276-8

DOI: $10.1007 /$ s11606-020-06466-x

(C) Society of General Internal Medicine 2021

\section{INTRODUCTION}

Patient portals provide access to health information, creating the conditions necessary for patient engagement. ${ }^{1}$ However, digital disparities limit engagement with portals. ${ }^{1}$ To reduce these disparities, some healthcare systems have expanded portal access to informal caregivers, like spouses, or family members. Patients - particularly those with low digital literacy or complex health needs - have expressed interest in sharing portal access with caregivers ${ }^{2,3}$; however, few healthcare systems enable patients to share portal access. ${ }^{4,5}$ Instead, some patients informally share log-on information with caregivers, creating challenges for healthcare systems in recognizing whether portal activity is from patients or caregivers. ${ }^{4}$ To date, there have been no nationally representative studies that estimate how often caregivers access portals or which caregivers are most likely to have access. Our study has two objectives ${ }^{1}$ : estimate the prevalence of portal usage among caregivers in a nationally representative sample and ${ }^{2}$ examine predictors of portal usage.

\section{METHODS}

Data were obtained from the 2019 Health Information National Trend Survey (HINTS) $(n=4200$ adults and 612 informal caregivers). HINTS measures portal usage for self-care and as part of caregiving activities in the past 12 months using separate questions. We included variables associated with portal usage including demographics and presence of a chronic or mental health condition. ${ }^{1}$ For caregivers, we also included relation to patient (e.g., child), and health condition of the patient.

We compared portal usage for self-care and for caregiving based on sample characteristics. We conducted two multivariable logistic regressions to examine which characteristics were associated with portal usage for self-care and caregiving. Statistical analyses were conducted in Stata (version 16). We applied sampling and jackknife replicate weights to develop

Received July 29, 2020

Accepted December 13, 2020

Published online January27, 2021 nationally representative estimates and account for complex survey design. This study was exempted by the Advarra Institutional Review Board.

\section{RESULTS}

The sample contained 4200 adults; 612 identified as informal caregivers (Table 1). Less than half of adults $(42.5 \%)$ used portals for self-care and less than a third of caregivers (31.4\%) used portals for caregiving. In multivariable analyses, individuals under 65 were more likely to use a portal for self-care (OR: $1.22, P=0.009$ ) and caregiving (OR: $1.77, P=0.03$ ) (Table 2). Having an income $>\$ 75,000$ annually was associated with higher odds of portal use for self-care (OR: 3.39, $P<$ 0.001 ) and caregiving (OR: $3.02, P=0.003)$ compared to having an income of $<\$ 20,000$ annually. Having a chronic health condition was associated with greater odds of portal use for self-care (OR: $1.33, P<0.001$ ) but not for caregiving. Having a mental health condition was associated with greater odds of portal use for self-care (OR: $1.23, P=0.01$ ) but reduced odds of portal use for caregiving (OR: 0.61, $P=$ 0.03). Caring for a patient with a chronic condition was associated with greater odds of portal use for caregiving (OR: $1.66, P=0.022$ ). Caring for a patient with a mental health condition was associated with greater odds of portal use for caregiving (OR: 1.71, $P=0.013$ ).

\section{DISCUSSION}

In the USA, 53 million adults serve as caregivers and help patients with tasks that could be completed through the patient portal (e.g., scheduling appointments, communicating with providers). ${ }^{6}$ Our study found that a third of caregivers use portals nationally, demonstrating interest in shared portal access. Prior studies suggest that granting caregiver access to portals can be cumbersome (e.g., lengthy credentialing process) and may not meet patients' needs (e.g., inability to align information sharing with patient preferences). ${ }^{4,7}$ Our study found that digital disparities hindered caregivers' access to patient portals; older caregivers and caregivers with fewer economic resources were less likely to use the portal. Race and ethnicity were not associated with caregiver portal use; however, further studies are needed to examine this relationship. Healthcare systems may need to develop iPad loan programs and digital literacy training that target both patients 
Table 1 Sample Characteristics

\begin{tabular}{|c|c|c|c|c|c|c|}
\hline \multirow[t]{4}{*}{ Characteristics } & \multicolumn{2}{|c|}{ Patients' portal use } & \multirow[t]{4}{*}{$P$} & \multicolumn{2}{|c|}{ Caregivers' portal use } & \multirow[t]{4}{*}{$P$} \\
\hline & All & Users & & All & Users & \\
\hline & $n=4200$ & $n=1785$ & & $n=612$ & $n=192$ & \\
\hline & $W=199696652$ & $W=81927907$ & & $W=29980581$ & $W=10148918$ & \\
\hline Age, mean (SD) & $55.8(16.6)$ & $54.1(16.0)$ & \multirow[t]{2}{*}{$<0.001$} & $54.7(14.3)$ & $52.0(14.2)$ & \multirow[t]{2}{*}{$<0.001$} \\
\hline Under $65, N(\%)$ & $2772(66.0)$ & $1249(70.0)$ & & $461(75.3)$ & $155(80.7)$ & \\
\hline Male gender, $N(\%)$ & $1818(43.3)$ & $703(38.7)$ & $<0.001$ & $223(36.4)$ & $62(32.3)$ & 0.07 \\
\hline Black race, $N(\%)$ & $706(16.8)$ & $238(33.7)$ & $<0.001$ & $112(18.3)$ & $30(15.6)$ & 0.20 \\
\hline Hispanic ethnicity, $N(\%)$ & $617(14.7)$ & $220(35.7)$ & $<0.001$ & $89(14.5)$ & $33(17.2)$ & 0.57 \\
\hline \multicolumn{7}{|l|}{ Education, $N(\%)$} \\
\hline High school diploma or less & 877 (20.9) & $674(31.7)$ & \multirow[t]{3}{*}{$<0.001$} & $270(44.1)$ & $68(35.4)$ & \multirow[t]{3}{*}{0.002} \\
\hline College degree & $1195(28.5)$ & $597(49.9)$ & & $184(30.1)$ & $65(33.9)$ & \\
\hline Postgraduate & $2128(50.7)$ & $514(58.6)$ & & $158(25.8)$ & $59(30.7)$ & \\
\hline \multicolumn{7}{|l|}{ Income, $N(\%)$} \\
\hline Less than $\$ 20,000$ & $718(17.1)$ & $149(20.8)$ & \multirow[t]{5}{*}{$<0.001$} & $83(13.6)$ & $14(7.3)$ & \multirow[t]{5}{*}{0.005} \\
\hline$\$ 20,000$ to $\$ 34,999$ & $521(12.4)$ & $161(30.9)$ & & $69(11.3)$ & $18(9.4)$ & \\
\hline$\$ 35,000$ to $\$ 49,999$ & $538(12.8)$ & $222(41.3)$ & & $76(12.4)$ & $24(12.5)$ & \\
\hline$\$ 50,000$ to $\$ 74,999$ & $755(18.0)$ & $353(46.8)$ & & $115(18.8)$ & $37(19.3)$ & \\
\hline$\$ 75,000$ or more & $1668(39.7)$ & $900(53.9)$ & & $269(44.0)$ & 99 (51.6) & \\
\hline Rural residence, $N(\%)$ & $449(10.7)$ & $152(33.9)$ & $<0.001$ & $63(10.3)$ & $16(8.3)$ & 0.18 \\
\hline Chronic condition, $N(\%)$ & $2284(54.4)$ & $955(41.8)$ & 0.33 & $212(34.6)$ & $82(42.7)$ & 0.02 \\
\hline Mental health condition, $N(\%)$ & $939(22.4)$ & $423(45.1)$ & 0.07 & $158(25.8)$ & $65(33.9)$ & 0.003 \\
\hline Caregiver chronic condition, $N(\%)$ & $\mathrm{n} / \mathrm{a}$ & $\mathrm{n} / \mathrm{a}$ & $\mathrm{n} / \mathrm{a}$ & $329(53.8)$ & $99(51.6)$ & 0.16 \\
\hline Caregiver mental condition, $N(\%)$ & $\mathrm{n} / \mathrm{a}$ & $\mathrm{n} / \mathrm{a}$ & $\mathrm{n} / \mathrm{a}$ & $171(27.9)$ & $43(22.4)$ & 0.06 \\
\hline \multicolumn{7}{|l|}{ Relation to patient, $N(\%)$} \\
\hline Child & $\mathrm{n} / \mathrm{a}$ & $\mathrm{n} / \mathrm{a}$ & $\mathrm{n} / \mathrm{a}$ & $155(25.4)$ & $53(27.6)$ & \multirow[t]{4}{*}{0.001} \\
\hline Spouse/partner & $\mathrm{n} / \mathrm{a}$ & $\mathrm{n} / \mathrm{a}$ & $\mathrm{n} / \mathrm{a}$ & $144(23.5)$ & $54(28.1)$ & \\
\hline Parent & $\mathrm{n} / \mathrm{a}$ & $\mathrm{n} / \mathrm{a}$ & $\mathrm{n} / \mathrm{a}$ & $206(33.7)$ & $61(31.8)$ & \\
\hline Friend or other family member & $\mathrm{n} / \mathrm{a}$ & $\mathrm{n} / \mathrm{a}$ & $\mathrm{n} / \mathrm{a}$ & $107(17.4)$ & $24(12.5)$ & \\
\hline
\end{tabular}

and caregivers to overcome digital disparities. Furthermore, implementation research is needed to determine best practices for caregiver proxy access (e.g., which patient information to share, what resources caregivers value). This study has several limitations. Data are self-report which may be prone to bias and were only available for 2019 so we cannot examine trends in portal use. Overall, our study found that many informal caregivers use patient portals for caregiving activities. Further research is needed to understand how healthcare systems can optimize the use of portals for caregiver and patient needs.

Table 2 Predictors of Portal Use

\begin{tabular}{|c|c|c|c|c|c|c|}
\hline & \multicolumn{2}{|c|}{ Patients' portal use } & & \multicolumn{2}{|c|}{ Caregivers' portal use } & \\
\hline & \multicolumn{3}{|l|}{$n=4200$} & \multicolumn{2}{|l|}{$n=612$} & \\
\hline & \multicolumn{3}{|c|}{$W=199696652$} & \multicolumn{3}{|c|}{$W=29980581$} \\
\hline & OR & $95 \% \mathrm{CI}$ & $P$ value & OR & $95 \% \mathrm{CI}$ & $P$ value \\
\hline Under 65 & 1.22 & $1.05-1.42$ & 0.009 & 1.77 & $1.07-2.94$ & 0.03 \\
\hline Male gender & 0.67 & $0.59-0.77$ & 0.04 & 0.66 & $0.45-0.98$ & 0.04 \\
\hline Black race & 0.77 & $0.64-0.93$ & 0.006 & 0.75 & $0.46-1.24$ & 0.26 \\
\hline Hispanic ethnicity & 0.80 & $0.66-0.96$ & 0.017 & 1.39 & $0.83-2.34$ & 0.21 \\
\hline \multicolumn{7}{|c|}{ Education } \\
\hline \multicolumn{7}{|c|}{ High school diploma or less (ref) } \\
\hline College degree & 1.63 & $1.29-2.06$ & $<0.001$ & 1.51 & $0.97-2.36$ & 0.07 \\
\hline Postgraduate & 2.23 & $1.72-2.89$ & $<0.001$ & 1.48 & $0.91-2.42$ & 0.12 \\
\hline \multicolumn{7}{|l|}{ Income } \\
\hline \multicolumn{7}{|l|}{ Less than $\$ 20,000$ (ref) } \\
\hline$\$ 20,000$ to $\$ 34,999$ & 1.62 & $1.24-2.11$ & 0.07 & 1.83 & $0.80-4.23$ & 0.15 \\
\hline$\$ 35,000$ to $\$ 49,999$ & 2.45 & $1.89-3.17$ & 0.002 & 2.13 & $0.96-4.73$ & 0.06 \\
\hline$\$ 50,000$ to $\$ 74,999$ & 2.89 & $2.28-3.69$ & 0.002 & 2.28 & $1.08-4.82$ & 0.03 \\
\hline$\$ 75,000$ or more & 3.39 & $2.70-4.27$ & $<0.001$ & 3.02 & $1.47-6.22$ & 0.003 \\
\hline Rural residence & 0.70 & $0.49-1.00$ & 0.003 & 0.83 & $0.43-1.60$ & 0.59 \\
\hline Chronic condition & 1.33 & $1.15-1.53$ & $<0.001$ & 2.01 & $1.32-3.07$ & 0.001 \\
\hline Mental health condition & 1.23 & $1.05-1.44$ & 0.01 & 1.99 & $1.31-3.03$ & 0.001 \\
\hline Caregiver chronic condition & $\mathrm{n} / \mathrm{a}$ & $\mathrm{n} / \mathrm{a}$ & $\mathrm{n} / \mathrm{a}$ & 1.08 & $0.74-1.58$ & 0.70 \\
\hline Caregiver mental condition & $\mathrm{n} / \mathrm{a}$ & $\mathrm{n} / \mathrm{a}$ & $\mathrm{n} / \mathrm{a}$ & 0.61 & $0.39-0.96$ & 0.03 \\
\hline \multicolumn{7}{|l|}{ Relation to patient } \\
\hline Child & $\mathrm{n} / \mathrm{a}$ & $\mathrm{n} / \mathrm{a}$ & $\mathrm{n} / \mathrm{a}$ & 1.31 & $0.75-2.29$ & 0.35 \\
\hline Spouse/partner & $\mathrm{n} / \mathrm{a}$ & $\mathrm{n} / \mathrm{a}$ & $\mathrm{n} / \mathrm{a}$ & 1.79 & $1.05-3.06$ & 0.03 \\
\hline Parent & $\mathrm{n} / \mathrm{a}$ & $\mathrm{n} / \mathrm{a}$ & $\mathrm{n} / \mathrm{a}$ & 0.68 & $0.39-1.19$ & 0.18 \\
\hline Friend/family member & $\mathrm{n} / \mathrm{a}$ & $\mathrm{n} / \mathrm{a}$ & $\mathrm{n} / \mathrm{a}$ & 0.62 & $0.33-1.15$ & 0.13 \\
\hline
\end{tabular}


Kea Turner, $P h D^{1}$

Grace Wei, $\mathrm{MPH}^{2}$

Amy K. Otto, $\mathrm{PhD}^{1}$

Maija Reblin, $\mathrm{PhD}^{1}$

${ }^{1}$ Department of Health Outcomes and Behavior, Moffitt Cancer Center,

Tampa, FL, USA

${ }^{2}$ University of South Florida Morsani College of Medicine,

Tampa, FL, USA

Corresponding Author: Kea Turner, PhD; Department of Health Outcomes and Behavior, Moffitt Cancer Center, Tampa, FL, USA (e-mail: kea.turner@moffitt.org).

\section{Compliance with Ethical Standards:}

Conflict of Interest: Dr. Otto reports grants from National Cancer Institute during the conduct of the study.

\section{REFERENCES}

1. Lyles CR, Nelson EC, Frampton S, Dykes PC, Cemballi AG, Sarkar U. Using Electronic Health Record Portals to Improve Patient Engagement: Research Priorities and Best Practices. Ann Intern Med. 2020;172(11_Supplement):S123-S9. https://doi.org/10.7326/M19-0876

2. Mayberry LS, Kripalani S, Rothman RL, Osborn CY. Bridging the Digital Divide in Diabetes: Family Support and Implications for Health Literacy. Diabetes Technol Ther. 2011;13(10):1005-12. doi:https://doi.org/10. 1089/dia.2011.0055

3. Reed ME, Huang $J$, Brand $R$, Ballard D, Yamin C, Hsu J, et al. Communicating Through a Patient Portal to Engage Family Care Partners. JAMA Intern Med. 2018;178(1):142-4. doi:https://doi.org/10.1001/ jamainternmed.2017.6325

4. Wolff JL, Berger A, Clarke D, Green JA, Stametz R, Yule C, et al. Patients, Care Partners, and Shared Access to the Patient Portal: Online Practices at an Integrated Health System. J Am Med Inform Assoc. 2016;23(6):1150-8. doi:https://doi.org/10.1093/jamia/ocw025

5. Semere W, Crossley S, Karter AJ, Lyles CR, Brown W, Reed M, et al. Secure Messaging with Physicians by Proxies for Patients with Diabetes: Findings from the ECLIPPSE Study. J Gen Intern Med. 2019;34(11):24906. doi:https://doi.org/10.1007/s11606-019-05259-1

6. NAC. Caregiving in the US. Washington D.C.2020.

7. Sarkar U, Bates DW. Care Partners and Online Patient Portals. JAMA. 2014;311(4):357-8. doi:https://doi.org/10.1001/jama.2013.285825

Publisher's Note: Springer Nature remains neutral with regard to jurisdictional claims in published maps and institutional affiliations. 